\title{
Political Orientation Predicts Credulity Regarding Putative Hazards
}

2017, Vol. 28(5) 651-660

(C) The Author(s) 2017

Reprints and permissions

sagepub.com/journalsPermissions.nav DOI: $10.1177 / 0956797617692108$

www.psychologicalscience.org/PS @SAGE

\author{
Daniel M. T. Fessler ${ }^{1,2}$, Anne C. Pisor ${ }^{3,4}$, and Colin Holbrook ${ }^{1,2}$ \\ ${ }^{1}$ Department of Anthropology, University of California, Los Angeles; ${ }^{2}$ Center for Behavior, Evolution, \& \\ Culture, University of California, Los Angeles; ${ }^{3}$ Department of Anthropology, University of California, \\ Santa Barbara; and ${ }^{4}$ Department of Human Behavior, Ecology, and Culture, Max Planck Institute for \\ Evolutionary Anthropology, Leipzig, Germany
}

\begin{abstract}
To benefit from information provided by other people, people must be somewhat credulous. However, credulity entails risks. The optimal level of credulity depends on the relative costs of believing misinformation and failing to attend to accurate information. When information concerns hazards, erroneous incredulity is often more costly than erroneous credulity, given that disregarding accurate warnings is more harmful than adopting unnecessary precautions. Because no equivalent asymmetry exists for information concerning benefits, people should generally be more credulous of hazard information than of benefit information. This adaptive negatively biased credulity is linked to negativity bias in general and is more prominent among people who believe the world to be more dangerous. Because both threat sensitivity and beliefs about the dangerousness of the world differ between conservatives and liberals, we predicted that conservatism would positively correlate with negatively biased credulity. Two online studies of Americans supported this prediction, potentially illuminating how politicians' alarmist claims affect different portions of the electorate.
\end{abstract}

\section{Keywords}

threat sensitivity, negativity bias, negatively biased credulity, political orientation, open data, open materials

Received 5/29/16; Revision accepted 1/14/17

In 2012, a liberal professor wrote that the Obama administration was stockpiling ammunition in preparation for totalitarian rule. This idea was ignored by liberals. In 2015, conservative bloggers asserted that the real goal of a military exercise was to occupy Texas and impose martial law. Conservatives became so concerned that the governor of Texas ordered the Texas State Guard to monitor the exercise.

The different fates of these two conspiracy theories might simply reflect their historical particulars. Whereas in 2012 liberal Americans largely approved of the Obama Administration, in 2015 most conservative Americans did not. Perhaps the first theory died while the second prospered simply because the latter resonated with the views of a substantial audience and the former did not. However, two bodies of research suggest that psychological differences related to political orientation may also have been at work. First, a sizeable literature documents that, in the United States, responsiveness to negative stimuli correlates with political orientation; conservatives display more responsiveness and liberals display less. Second, recent studies indicate that people are more credulous about information concerning hazards than about information concerning benefits-and individuals differ in this regard. In the current study, we combined these approaches, testing the hypothesis that political orientation is correlated with differences in credulity toward hazard information. If correct, this thesis potentially illuminates the differential effects of politicians' alarmist claims on liberal and conservative constituencies.

We use the terms "liberal" and "conservative" recognizing that these are heterogeneous categories and that

\section{Corresponding Author:}

Daniel M. T. Fessler, Department of Anthropology, 341 Haines Hall, University of California, Los Angeles, Los Angeles, CA 90095-1553

E-mail: dfessler@anthro.ucla.edu 
self-identifying members of each may hold internally incompatible positions on various issues. We view these inconsistencies as a source of noise; hence, any differences found despite these features constitute foundational orientations shared by core groups of category members (Weeden \& Kurzban, 2016). Research has revealed psychological differences between liberals and conservatives, including both broad features of personality (Carney, Jost, Gosling, \& Potter, 2008) and the priority given to different moral principles (Graham, Haidt, \& Nosek, 2009). Reviewing a large number of studies, Hibbing, Smith, and Alford (2014) concluded that conservatives display greater negativity bias than do liberals-or, perhaps more precisely, threat bias (Lilienfeld \& Latzman, 2014), or sensitivity to the possibility of danger. Subsequent research has largely bolstered this conclusion (Ahn et al., 2014; Mills et al., 2016; Mills, Smith, Hibbing, \& Dodd, 2014; but see Knoll, O’Daniel, \& Cusato, 2015).

Like other animals, humans exhibit negativity biascompared with positive events, negative events capture attention and direct information processing more readily, elicit strong emotions more easily, and are more memorable (Baumeister, Bratslavsky, Finkenauer, \& Vohs, 2001; Rozin \& Royzman, 2001). Valence may reflect the implications for biological fitness (survival and subsequent reproductive success) that a class of events would have had in ancestral environments. Hence, negativity bias plausibly stems from the detrimental fitness consequences of failing to immediately attend to, address, and learn from fitness-reducing events; such consequences will generally be greater than those of failing to do so for fitnessenhancing events, given that threats frequently are more imminent than, and preclude, opportunities (Baumeister et al., 2001; Rozin \& Royzman, 2001). Within a species, the optimal level of negativity bias will depend on the interaction of features of both the individual and the environment (e.g., a vulnerable individual in a hazardous environment should be guided by greater negativity bias than a robust individual in a safe environment): There is no invariantly "correct" degree of negativity bias, and, correspondingly, natural selection has preserved substantial individual differences in negativity bias. If a core dimension of political orientation is that liberals value the opportunities afforded by change and cultural heterogeneity, whereas conservatives value the safety of tradition and cultural homogeneity, then conservatism is more consonant with pronounced negativity bias than is liberalism: Conservatives will often see pitfalls where liberals see promise (Hibbing et al., 2014).

Although the evolutionary considerations underlying negativity bias apply across species, in humans they intersect with a reliance on cultural information. Humans uniquely exploit cumulative cultural evolution and the technological and organizational advantages that it provides-humans are culture-dependent, a characteristic likely undergirded by specific psychological mechanisms for acquiring cultural information (Fessler, 2006). Relying on cultural information necessitates credulity: The utility of a given practice is frequently not evident to the learner, and is often opaque even to teachers (Boyd \& Richerson, 2006). However, people who are overly credulous risk acquiring erroneous information and being exploited (Kurzban, 2007). The trade-off between the benefits of credulity and its costs varies as a function of information type, such that the optimal level of credulity differs for different messages. With regard to information concerning hazards, the costs of erroneous credulity will often be lower than the costs of erroneous incredulity; although the former results in unnecessary precautions, the latter can result in injury or death. (As these possibilities indicate, the extent of the asymmetry in costs depends on the magnitude of the consequences should the information prove accurate.) Because no equivalently overarching asymmetry exists with regard to information concerning benefits, people should exhibit negatively biased credulity; that is, all other things being equal, people should more readily view as true information concerning hazards relative to information concerning benefits (Fessler, Pisor, \& Navarrete, 2014). Experimental results confirm this-when statements are framed as being about hazards, they are judged more likely to be true than when they are framed as involving benefits (Fessler, Pisor, \& Navarrete, 2014; see also Hilbig, 2009, 2012a, 2012b).

At the proximate level of explanation, negatively biased credulity is accounted for by the greater processing fluency attending negative information relative to positive information, thus linking negatively biased credulity to negativity bias in general (Hilbig, 2009, 2012a, 2012b). Given that conservatives display greater threat sensitivity and perhaps greater negativity bias than do liberals, this proximate pathway generates the prediction that conservatives will exhibit greater negatively biased credulity than will liberals. This prediction is reinforced by additional conceptual and empirical considerations.

Because newly identified hazards often share features (and therefore co-occur) with previously known hazards, the more dangerous the world in which one lives, the more likely it is that one will encounter additional hazards, and thus the greater the asymmetry between the costs of erroneous credulity and those of erroneous incredulity when assessing information concerning hazards. Accordingly, individuals who know (or believe they know) of the existence of many hazards should display elevated negatively biased credulity. This functionality is reinforced at the proximate level, because congruence between a message and prior beliefs enhances biased credulity (White, Pahl, Buehner, \& Haye, 2003). Accordingly, belief that the world is dangerous correlates 
positively with negatively biased credulity (Fessler, Pisor, \& Navarrete, 2014). In keeping with conservatives' view of tradition and cultural homogeneity as buffers against an uncertain world, conservatism is linked with beliefs in a dangerous world, both directly and via associations with authoritarianism (Federico, Hunt, \& Ergun, 2009; relatedly, see Altemeyer, 1998; Crowson, Thoma, \& Hestevold, 2005; Duckitt, 2001; Duckitt, Wagner, du Plessis, \& Birum, 2002; Lilienfeld \& Latzman, 2014; van Leeuwen \& Park, 2009). Hence, if conservatives view the world as more dangerous than do liberals, then conservatives should display more negatively biased credulity than liberals. To test this prediction, we measured negatively biased credulity and assessed political orientation in two U.S. samples.

\section{Study 1 \\ Metbod}

Participants. Given the variance observed in Study 2 of Fessler, Pisor, and Navarrete (2014), a final sample size of 450 was targeted. Expecting attrition and exclusions, in early October of 2015, we recruited 540 U.S. participants via Amazon's Mechanical Turk. Each participant was paid $\$ 0.50$. Data were prescreened for minimal completeness (see below), repeat participation, a study-completion time of at least 3 min, participants' first language being English, and correct responses to catch questions (descriptive statistics appear in Table S2a and predictors of exclusion are reported in Table S3, both in the Supplemental Material available online). The final sample consisted of 472 adults ( $48 \%$ female; $81 \%$ White) ranging in age from 19 to 65 years (mean age $=36.03$ years, $S D=11.81$ ).

Materials and procedure. We created a credulity assessment consisting of 14 plausible but false statements and 2 true statements; the latter were included so that we could honestly inform participants that some of the statements were factual. These 16 statements addressed eight domains; for each domain, 1 statement concerned a benefit and 1 concerned a hazard (e.g., "Eating carrots results in significantly improved vision" and "Kale contains thallium, a toxic heavy metal, that the plant absorbs from soil"; for the complete instrument, see the Supplemental Material). Participants reported judgments of truthfulness on a scale from 1 to $7(1=I$ 'm absolutely certain this statement is FALSE, 7 = I'm absolutely certain this statement is TRUE). As noted earlier, the magnitude of the hazard or benefit addressed by a statement should color credulity toward it, because any asymmetry between the costs of erroneous credulity and erroneous incredulity will be a function of the significance of the benefit or hazard at issue. Statements were therefore selected so that, for a given domain, the presumed magnitudes of the benefit or hazard were approximately equal; in addition, participants were asked to judge these magnitudes on a scale from 1 to 7 ( 1 = The benefit [hazard] described in this statement is SMALL, $7=$ The benefit [hazard] described in this statement is LARGE).

In cases of incomplete responses, if a participant left fewer than $10 \%$ of the items unanswered, missing responses were imputed (see Appendix S3 in the Supplemental Material; for model fits without imputation, see Table $\mathrm{S} 5$ in the Supplemental Material). Statements were presented in truly random order. To measure bias in credulity regarding hazard information relative to benefit information, in the models reported in the main text, we examined the difference between hazard credulity and benefit credulity; the Supplemental Material presents complementary models that examined only hazard credulity or only benefit credulity as the response (Tables S6a and S6b).

Next, political orientation was assessed using four measures: First, participants completed a slightly updated form of the Dodd et al. (2012) issues index, which employs the Wilson-Patterson (1968) format (see the Supplemental Material), in which participants indicated whether they agreed with, disagreed with, or were uncertain regarding 28 contemporary issues, half of which are favored by conservatives (e.g., "Biblical truth," "tax cuts") and half of which are favored by liberals (e.g., "abortion rights," "socialism"). For each conservative topic, agreement was scored as +1 and disagreement as -1 ; responses to the liberal topics were reverse-scored; "uncertain" was scored as 0. With three exceptions (see Appendix S1 in the Supplemental Material), responses to all topics were summed such that increasingly positive values indicated greater conservatism $(\alpha=.88)$.

Second, participants completed the Dodd et al. (2012) social-principles index (minus one item concerning danger; see the Supplemental Material). They selected one of two completions of the stem "Society works best when ..."; for example: "people are rewarded according to merit" or "people are rewarded according to need." The choices were intended to capture preferences for traditional social order, in-group favoritism, obedience to authority, and punishment of transgressions. Typically conservative responses were coded as 1, typically liberal responses were coded as -1 , and then responses were summed such that larger values indicated greater conservatism $(\alpha=.72)$. All items and stem-completion options were presented in truly random order. For each of the measures taken from Dodd et al., any missing values were imputed if participants answered at least $90 \%$ of that measure's items (for fit without imputation, see Table S5 in the Supplemental Material). 
Third, participants indicated their political position on a 9-point scale ( 1 = strongly liberal, 9 = strongly conservative).

Finally, participants reported their political-party affiliation, scored as +1 for traditionally conservative parties (Republican, Tea Party), -1 for traditionally liberal parties (Democrat, Green), and 0 for Libertarians or unaffiliated people. Demographic items followed, including parenthood status; our previous research (Fessler, Holbrook, Pollack, \& Hahn-Holbrook, 2014) suggests that parents may be more sensitive to the presence of hazards than nonparents. In addition, participant height and selfassessed fighting ability were collected for a future study; exploratory analyses indicate that these had no bearing on the results of interest here, and so they are not reported. For the complete survey, see the Supplemental Material.

\section{Results}

To facilitate participant comprehension in our credulity measure, we anchored the low end of the Likert-type scale at 1 (I'm absolutely certain this statement is FALSE). Our weighting procedure involved multiplying the participant's response on this scale by the participant's assessment of the magnitude of the given hazard or benefit. Accordingly, to preclude assigning a positive multiplicative product to items deemed entirely false by a participant, we began by subtracting 1 from all credulity responses.

Because our four measures of political orientation had disparate ranges, we $z$-scored each measure, performed a principal components analysis, and extracted the first component (this component summarized $72.65 \%$ of the variance, and each measure had a loading of .80 or higher). The result was used as a summary measure of political orientation, higher values indicating greater conservatism. (An alternative variable created by summing the four measures together produced similar results when included in our models—-see Table S7 in the Supplemental Material.)

Using the $\mathrm{R}$ software environment (Version 3.3.1; R Development Core Team, 2016), we fit linear models, with the difference between hazard credulity (weighted by the participant's perceived magnitude for each respective item) and benefit credulity (similarly weighted) as the response. Variables that exhibited negative skewness were rounded down to the 97.5th percentile, and those that exhibited positive skewness were rounded up to the 2.5th percentile (see Appendix S2 in the Supplemental Material). No models exhibited collinearity (i.e., a variance inflation factor greater than 3).

The participants' tendency, albeit nonsignificant, was to find our (almost entirely false) weighted credulityassessment items more believable if they concerned a hazard rather than a benefit (hazard: $M=12.28$; benefit: $M=11.96), t(934.51)=1.02, p=.31$. Participants' average credulity toward benefits was correlated with their average credulity toward hazards, $r=.41$.

Addressing the key prediction at issue, we found that participants who were more conservative were significantly more likely to exhibit greater credulity for information about hazards relative to information about benefits (Table 1), an effect that was independent of the effects of controls (see Table S4 in the Supplemental Material).

Table 1. Results From Models Using the Summary Measure of Political Orientation to Predict the Difference Between Weighted Hazard Credulity and Weighted Benefit Credulity

\begin{tabular}{|c|c|c|c|c|}
\hline \multirow[b]{2}{*}{ Study and predictor } & \multicolumn{2}{|c|}{$\begin{array}{l}\text { Parameter } \\
\text { estimate }\end{array}$} & \multirow{2}{*}{$\begin{array}{c}95 \% \\
\text { confidence } \\
\text { interval for } b\end{array}$} & \multirow[b]{2}{*}{$p$} \\
\hline & $b$ & $\beta$ & & \\
\hline \multicolumn{5}{|l|}{ Study $1(N=472)^{\mathrm{a}}$} \\
\hline Intercept & -0.27 & 0.00 & {$[-2.09,1.54]$} & .77 \\
\hline Political orientation summary & 0.36 & 0.12 & {$[0.08,0.65]$} & .01 \\
\hline \multicolumn{5}{|l|}{ Study $2(N=476)^{\mathrm{b}}$} \\
\hline Intercept & 0.63 & 0.00 & {$[-1.25,2.52]$} & .51 \\
\hline Political orientation summary & 0.54 & 0.19 & {$[0.28,0.81]$} & .00 \\
\hline
\end{tabular}

${ }^{a}$ Model-fit statistics for Study 1: adjusted $R^{2}=.01, F(10,461)=1.66, p=.09$. Women, people who did not self-identify as "White," people with some high school education or a high school diploma, and nonparents are the contrast levels included in the intercept. Age was centered such that the intercept represents age 19. ${ }^{\mathrm{b}}$ Model-fit statistics for Study 2: adjusted $R^{2}=.03, F(12,463)=2.09, p=.02$. Women, people who did not self-identify as "White," people with some high school education or a high school diploma, and people with median general reasoning ability are the contrast levels included in the intercept. Age was centered such that the intercept represents age 18. Parenthood status was excluded in this model because of the large number of incomplete responses to demographic items concerning parenthood (for regression results for the subset of Study 2 participants for whom parenthood status was available, see Table S10 in the Supplemental Material). 
Table 2. Results From Study $1(N=472)$ : Models Using the Distinct Political Measures to Predict the Difference Between Weighted Hazard Credulity and Weighted Benefit Credulity

\begin{tabular}{|c|c|c|c|c|}
\hline \multirow[b]{2}{*}{ Model and predictor } & \multicolumn{2}{|c|}{$\begin{array}{c}\text { Parameter } \\
\text { estimate }\end{array}$} & \multirow{2}{*}{$\begin{array}{l}95 \% \\
\text { confidence } \\
\text { interval for } b\end{array}$} & \multirow[b]{2}{*}{$p$} \\
\hline & $b$ & $\beta$ & & \\
\hline \multicolumn{5}{|l|}{ Issues model } \\
\hline Intercept & 0.07 & 0.00 & {$[-1.76,1.90]$} & .94 \\
\hline Dodd et al. issues-index score & 0.09 & 0.16 & {$[0.04,0.14]$} & .00 \\
\hline \multicolumn{5}{|l|}{ Social-principles model } \\
\hline Intercept & 0.02 & 0.00 & {$[-1.85,1.89]$} & .98 \\
\hline Dodd et al. social-principles index score & 0.09 & 0.09 & {$[0.00,0.18]$} & .05 \\
\hline \multicolumn{5}{|l|}{ Political-position model } \\
\hline Intercept & -1.00 & 0.00 & {$[-2.95,0.95]$} & .32 \\
\hline Political position & 0.17 & 0.07 & {$[-0.05,0.39]$} & .13 \\
\hline \multicolumn{5}{|l|}{ Political-affiliation model } \\
\hline Intercept & -0.81 & - & {$[-2.69,1.07]$} & .40 \\
\hline Libertarian or unaffiliated & 0.74 & - & {$[-0.34,1.83]$} & .18 \\
\hline Conservative & 0.97 & - & {$[-0.34,2.27]$} & .15 \\
\hline
\end{tabular}

Note: The issues model was the best-fitting model, adjusted $R^{2}=.02, F(10,461)=2.14, p=.02$. The statistics for the other models were as follows-social-principles model: adjusted $R^{2}=.01, F(10$, $461)=1.43, p=.17$; political-position model: adjusted $R^{2}=.01, F(10,461)=1.27, p=.25$; politicalaffiliation model: adjusted $R^{2}=.01, F(11,460)=1.20, p=.28$.

Treating hazard credulity separately from benefit credulity confirmed these results: Conservatism has a positive effect on hazard credulity but no effect on benefit credulity (see Tables S6a and S6b in the Supplemental Material). This was true even if we did not weight credulity by the participant's perceived magnitude of the hazard or benefit described in each item (see Table 58 in the Supplemental Material) or if we treated credulity for each item as a separate response (and included a random intercept for each participant and item; see Tables S9a and $\mathrm{S} 9 \mathrm{~b}$ in the Supplemental Material). Likewise, this effect was robust to the exclusion of any single item (see Fig. S1 in the Supplemental Material). The relationship between conservatism and negatively biased credulity was driven predominantly by participants' responses to the Dodd et al. (2012) issues index, which explained more variance in credulity than did the social principles index, participants' self-identified political position, or participants' self-identified political party (Table 2). More specifically, items from the issues index that addressed social conservatism predicted negatively biased credulity; items on conservative views on the military, on obedience to authority, and on punishment (hereafter called military conservatism) also predicted negatively biased credulity, albeit not significantly. However, items addressing fiscal conservatism had no effect on negatively biased credulity (Table 3 and Fig. 1a; for the items from the Dodd et al., 2012, issues index in each category, see Appendix S1 in the Supplemental Material).

\section{Discussion}

In Study 1, we documented the predicted association between political orientation and negatively biased credulity. However, probably because of the shortcomings of Mechanical Turk, the sample suffered nontrivial data loss and was not balanced regarding political orientation (i.e., it was skewed left). We therefore conducted a second study, recruiting participants via Prolific Academic, an online platform that is arguably superior to Mechanical Turk (Peer, Samat, Brandimarte, \& Acquisti, 2015). Study 2 also improved on Study 1 in that we replaced outdated military items ("Patriot Act," "Iraq war") with contemporary topics (e.g., "Drone strikes," "Bomb cities controlled by terrorists"). To rule out the possibility that the pattern documented in Study 1 derived from differences in general reasoning abilities (Kemmelmeier, 2008), we added short measures of problem-solving and abstract reasoning (see the Supplemental Material).

\section{Study 2}

\section{Method}

Participants. In early September of 2016, we recruited 738 U.S. participants via Prolific Academic. Each participant was paid $\$ 2.31$. Data were prescreened for completeness, repeat participation, a study-completion time of at least $10 \mathrm{~min}$ (the cutoff was extended from Study 1 due to 
Table 3. Results From Models Using Social Conservatism, Fiscal Conservatism, and Military Conservatism to Predict the Difference Between Weighted Hazard Credulity and Weighted Benefit Credulity

\begin{tabular}{lccccc}
\hline & \multicolumn{2}{c}{$\begin{array}{c}\text { Parameter } \\
\text { estimate }\end{array}$} & $\begin{array}{c}\text { 95\% } \\
\text { confidence }\end{array}$ & \\
\cline { 2 - 3 } Study and predictor & $b$ & $\beta$ & & interval for $b$ & $p$ \\
\hline Study 1 $(N=472)^{\mathrm{a}}$ & & & & & \\
$\quad$ Intercept & -0.44 & 0.00 & {$[-2.25,1.37]$} & .63 \\
Social conservatism & 0.29 & 0.11 & {$[0.00,0.58]$} & .05 \\
Fiscal conservatism & 0.00 & 0.00 & {$[-0.38,0.38]$} & .99 \\
Military conservatism & 0.23 & 0.07 & {$[-0.10,0.56]$} & .18 \\
Study 2 $(N=476)^{\mathrm{b}}$ & & & & .60 \\
$\quad$ Intercept & 0.51 & 0.00 & {$[-1.38,2.41]$} & .60 \\
Social conservatism & 0.33 & 0.14 & {$[0.07,0.59]$} & .01 \\
$\quad$ Fiscal conservatism & 0.13 & 0.04 & {$[-0.22,0.48]$} & .46 \\
$\quad$ Military conservatism & 0.24 & 0.09 & {$[-0.05,0.53]$} & .11 \\
\hline
\end{tabular}

Note: Each of the three subscales—social, fiscal, and military conservatism-was summarized by the first principal component; the three parameters reported for each model in the table are estimates for the effects of these three components on negatively biased credulity (for details, see the Supplemental Material).

${ }^{a}$ For the Study 1 model, adjusted $R^{2}=.02, F(12,459)=1.78, p=.046 .{ }^{b}$ For the Study 2 model, adjusted $R^{2}=.04, F(14,461)=2.24, p=.006$.

the addition of time-consuming measures of reasoning and problem solving), participants' first language being English, and correct responses to "catch questions" (descriptive statistics appear in Table S2b and predictors of exclusion are reported in Table S3, both in the Supplemental Material). Because the sample evinced a left-skewed political orientation, we randomly excluded participants who self-identified as more liberal (i.e., a 2 or lower on the 9-point political orientation scale) until our sample approximated the distribution of political orientations in the United States as documented in a Gallup poll conducted a few months before our study (Jones, 2016). Results are robust to the exclusion or inclusion of these individuals (see Table S11 in the Supplemental Material). The final sample consisted of 476 adults (40\% female; $79 \%$ White) ranging in age from 18 to $73(M=34.32, S D=12.56)$.
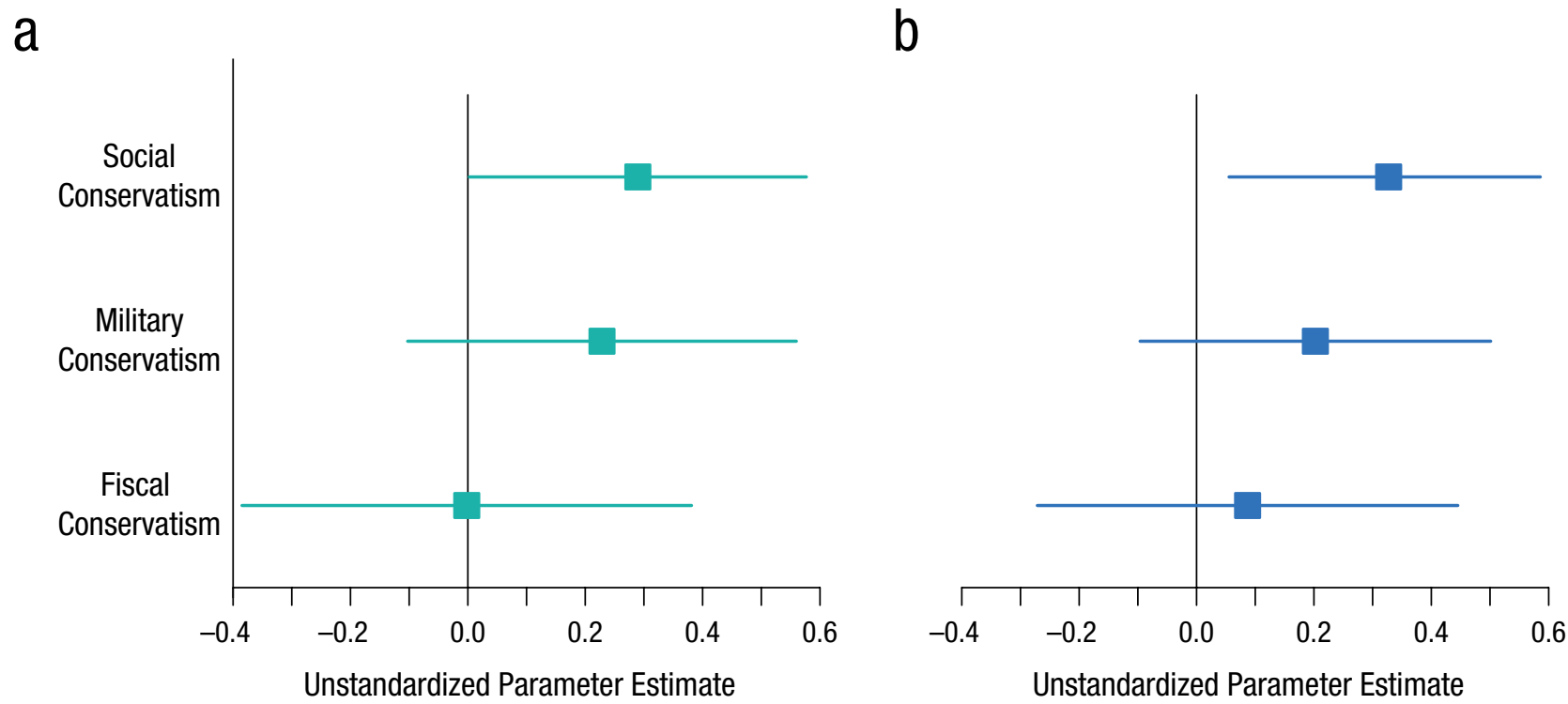

Fig. 1. Unstandardized parameter estimates for social, military, and fiscal conservatism as predictors of negatively biased credulity in (a) Study 1 and (b) Study 2. Error bars represent 95\% confidence intervals. 
Materials and procedure. Participants were presented with the same credulity scale as in Study 1 . Statements were presented in truly random order. Political orientation was assessed using the four measures described in Study 1. With two exceptions (see Appendix S1 in the Supplemental Material), responses to all topics in our modified version of the Dodd et al. (2012) issues index were summed; the scale had a high degree of internal consistency $(\alpha=.82)$. The Dodd et al. (2012) socialprinciples index (minus one item concerning dangersee the Supplemental Material) again had high internal consistency $(\alpha=.74)$. These indices were followed by demographic items and measures of general reasoning ability (for the complete survey, see the Supplemental Material). Many participants failed to indicate whether they were parents, so parenthood status was excluded from all models unless otherwise stated.

\section{Results}

Because our four measures of political orientation had disparate ranges, we $z$-scored each measure, performed a principal components analysis, and extracted the first component (this component summarized $73.90 \%$ of the variance, and each measure had a loading of .77 or higher). The result was used as a summary of political orientation, higher values indicating greater conservatism. (An alternative variable created by summing the four measures together produced similar results when included in our models-see Table S6 in the Supplemental Material.)

Linear models were fit with the difference between weighted hazard credulity and weighted benefit credulity as the response. Variables that exhibited negative skewness were rounded down to the 97.5 th percentile, and those that exhibited positive skewness were rounded up to the 2.5th percentile (see Appendix S2 in the Supplemental Material). No models exhibited collinearity (i.e., a variance inflation factor greater than 3).

Participants found weighted credulity-scale items significantly more believable if they concerned a hazard rather than a benefit (hazard: $M=12.82$; benefit: $M=$ $11.48), t=4.03, p<.001)$. Participants' average credulity toward benefits was correlated with their average credulity toward hazards, $r=.48$.

Addressing the key prediction at issue, participants who were more conservative were again significantly more likely to exhibit greater credulity for information about hazards relative to information about benefits (Table 1), an effect that was independent of the effects of controls (see Table S4 in the Supplemental Material); the same was true of the entire sample (i.e., when no highly liberal individuals are excluded-see Table S11 in the Supplemental Material). One item (concerning terrorism) had a large influence on hazard credulity. Although exclusion of this item diminished the magnitude of the effect so that it was no longer significant, the direction of the effect did not change across multiple iterations of the model; it varied only slightly as a function of the set of liberals excluded (see Fig. S1b in the Supplemental Material). Treating hazard credulity separately from benefit credulity corroborated the predicted relationship: Conservatism had a positive effect on hazard credulity but no effect on benefit credulity (see Tables S6a and S6b in the Supplemental Material; for the varied effect of excluding the terrorism item, see Fig. S2b in the Supplemental Material); this was true even if we did not weight credulity by the participant's perceived magnitude of the hazard or benefit described in each item (see Table S8 in the Supplemental Material) or if we treated credulity for each item as a separate response (and included a random intercept for each participant and item; see Tables S9a and S9b in the Supplemental Material).

As in Study 1, the relationship between conservatism and negatively biased credulity was driven predominantly by participants' responses to the Dodd et al. (2012) issues index rather than by their score on the Dodd et al. social-principles index, their self-identified political position, or their self-identified political party (Table 4). Also as in Study 1, items from the Dodd et al. issues index that addressed social conservatism predicted negatively biased credulity; once again, military conservatism also predicted negatively biased credulity, albeit not significantly. Fiscal conservatism again made no notable contribution in this regard (Table 3 and Fig. 1b; for the items from the Dodd et al. issues index in each category, see Appendix S1 in the Supplemental Material).

\section{General Discussion}

Because liberals and conservatives differ in their responsiveness to negative information, particularly concerning threats, and similarly differ in how dangerous they perceive the world to be, we predicted, and found, that political orientation correlated with the magnitude of the tendency to believe information about hazards more than information about benefits; liberals displayed less of this propensity and conservatives displayed more of it. This effect was driven by political orientation as defined by views on social issues. These results contribute to a corpus suggesting that, because of the intersection of variance in environments and variance in individual capabilities, a variety of potentially viable strategies emerge. Some individuals are more sensitive to the possibility of threats, and correspondingly pay higher precautionary costs; other people are less sensitive to this possibility, and pay higher costs when hazards are encountered.

Although the predicted relationships are evident in our results, these findings should be considered preliminary given that our samples were not representative nationwide samples and that our credulity measure 
Table 4. Results From Study $2(N=476)$ : Models With Distinct Political Measures as Predictors of the Difference Between Weighted Hazard Credulity and Weighted Benefit Credulity

\begin{tabular}{|c|c|c|c|c|}
\hline \multirow[b]{2}{*}{ Model and predictor } & \multicolumn{2}{|c|}{$\begin{array}{l}\text { Parameter } \\
\text { estimate }\end{array}$} & \multirow{2}{*}{$\begin{array}{c}95 \% \\
\text { confidence } \\
\text { interval for } b\end{array}$} & \multirow[b]{2}{*}{$p$} \\
\hline & $b$ & $\beta$ & & \\
\hline \multicolumn{5}{|l|}{ Issues model } \\
\hline Intercept & 0.89 & 0.00 & {$[-0.99,2.77]$} & .35 \\
\hline Dodd et al. issues-index score & 0.10 & 0.22 & {$[0.06,0.15]$} & .00 \\
\hline \multicolumn{5}{|l|}{ Social-principles model } \\
\hline Intercept & 0.82 & 0.00 & {$[-1.09,2.74]$} & .40 \\
\hline Dodd et al. social-principles index score & 0.12 & 0.15 & {$[0.05,0.20]$} & .00 \\
\hline \multicolumn{5}{|l|}{ Political-position model } \\
\hline Intercept & -1.03 & 0.00 & {$[-3.11,1.06]$} & .34 \\
\hline Political position & 0.33 & 0.14 & {$[0.11,0.54]$} & .00 \\
\hline \multicolumn{5}{|l|}{ Political-affiliation model } \\
\hline Intercept & -0.35 & - & {$[-2.33,1.64]$} & .73 \\
\hline Libertarian or unaffiliated & 0.77 & - & {$[-0.31,1.84]$} & .16 \\
\hline Conservative & 1.74 & - & {$[0.60,2.88]$} & .00 \\
\hline
\end{tabular}

consisted of a small number of items. Indeed, the limited scope of our instrument probably explains why the overarching pattern of negatively biased credulity, previously documented by Fessler, Pisor, and Navarrete (2014) using a different measure, was significant in Study 2 but not Study 1 . The same limitation may account for the outsized influence of one item on the key results of Study 2 .

Because older individuals display less negativity bias than younger individuals (Reed, Chan, \& Mikels, 2014), yet are generally more conservative (Cornelis, Van Hiel, Roets, \& Kossowska, 2009), some researchers have questioned the relationship between negativity bias and conservatism (Sedek, Kossowska, \& Rydzewska, 2014). Although our data did not resolve this question, we found no interaction between political orientation and age in predicting negatively biased credulity across wide age ranges (see Table S13 and Fig. S2 in the Supplemental Material). Rather, we found an effect of political orientation even when we controlled for age (Tables 1-4; see Table S4 in the Supplemental Material), which suggests independent effects.

In our results, social conservatism, but not fiscal conservatism, predicted increased negatively biased credulity. Whereas fiscal conservatism is orthogonal to individuals' exposure to hazards, adherence to what are seen as tried-and-true rules for social organization and personal comportment-the foundations of social conservatism-is, for its proponents, a defense against disorder and danger. Likewise, social conservatism correlates with threat-relevant personality features differentiating liberals and conservatives, but fiscal conservatism does not (Carney et al., 2008). Like social conservatism, conservative views on the military, on obedience to authority, and on endorsement of punishment address avenues for enhancing stability and safety. Consequently, what we have termed military conservatism can be expected to be associated with negatively biased credulity. Although in our models military conservatism did not significantly predict negatively biased credulity, the magnitude of the association between military conservatism and negatively biased credulity does not differ greatly from that of social conservatism (see Fig. 1). Future work should therefore further examine how military conservatism affects negatively biased credulity.

The difference in negatively biased credulity documented in this work likely interlocks with related phenomena. People judge individuals providing information about hazards to be more competent than individuals providing other information (Boyer \& Parren, 2015), a phenomenon consonant with negatively biased credulity; our findings suggest that conservatives will display this pattern more than will liberals. A parallel bias exists in information transmission, in that people are more likely to transmit messages concerning hazards than messages concerning benefits (Altshteyn, 2014; Bebbington, MacLeod, Ellison, \& Fay, in press; but see Stubbersfield, Tehrani, \& Flynn, 2015). Political orientation probably shapes this bias as well, which potentially influences the speed and breadth of dissemination of messages as a function of the political composition of a social network. A variety of phenomena 
thus link to negatively biased credulity in a manner suggesting that politicians' alarmist claims will affect liberals and conservatives differently.

In the 2016 U.S. election, then-candidate Donald Trump enjoyed support from social conservatives despite being a recent convert to their positions, despite displaying limited familiarity with their scriptures, and despite having boasted of violating one of their commandments. Although this support may have largely derived from, for example, President Trump's opposition to abortion, the relationship between political orientation and negatively biased credulity suggests that social conservatives may also have been influenced by his alarmist rhetoric, finding plausible such readily falsifiable claims as his tweet claiming that "inner-city crime is reaching record levels" (Trump, 2016). Likewise, although it is difficult to gauge the effect of fake news on the election, the credence given by social conservatives to bogus reports of nefarious conspiracies apparently explains why profit-minded purveyors of fake news disproportionately targeted conservative audiences (Sydell, 2016). More broadly, although distinguishing between Chicken Little and Cassandra is frequently difficult—with grave perils attending mistakes on both sides-it seems that social conservatives may be more apt to follow the former into the fox's den than they are to disregard the latter and witness the fall of Troy.

\section{Action Editor}

Eddie Harmon-Jones served as action editor for this article.

\section{Author Contributions}

D. M. T. Fessler and C. Holbrook conceived of the study. D. M. T. Fessler developed the methods with input from A. C. Pisor and C. Holbrook. C. Holbrook oversaw data collection. A. C. Pisor conducted all analyses, with input from C. Holbrook and D. M. T. Fessler. D. M. T. Fessler drafted the manuscript, and A. C. Pisor and C. Holbrook provided critical revisions. All the authors approved the final version of the manuscript for submission.

\section{Declaration of Conflicting Interests}

The authors declared that they had no conflicts of interest with respect to their authorship or the publication of this article.

\section{Funding}

C. Holbrook was supported by U.S. Air Force Office of Scientific Research Award FA9550-115-1-0469.

\section{Supplemental Material}

Additional supporting information can be found at http:// journals.sagepub.com/doi/suppl/10.1177/0956797617692108

\section{Open Practices

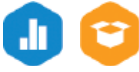

All data and materials have been made publicly available via the Open Science Framework and can be accessed at http://osf .io/qqq82. The data and materials are also publicly available via the University of California's eScholarship Web site and can be accessed at https://escholarship.org/uc/item/82j5p9r3. The complete Open Practices Disclosure for this article can be found at http://journals.sagepub.com/doi/suppl/10.1177/0956797617 692108. This article has received badges for Open Data and Open Materials. More information about the Open Practices badges can be found at http://www.psychologicalscience.org/ publications/badges.

\section{References}

Ahn, W.-Y., Kishida, K. T., Gu, X., Lohrenz, T., Harvey, A., Alford, J. R., . . . Dayan, P. (2014). Nonpolitical images evoke neural predictors of political ideology. Current Biology, 24, 2693-2699. doi:10.1016/j.cub.2014.09.050

Altemeyer, B. (1998). The other "authoritarian personality." In M. P. Zanna (Ed.), Advances in experimental social psychology (Vol. 30, pp. 47-92). San Diego, CA: Academic Press.

Altshteyn, I. (2014). Evidence for a warning bias in information transmission in social networks (Unpublished master's thesis). University of California, Los Angeles.

Baumeister, R. F., Bratslavsky, E., Finkenauer, C., \& Vohs, K. D. (2001). Bad is stronger than good. Review of General Psychology, 5, 323-370. doi:10.1037/1089-2680.5.4.323

Bebbington, K., MacLeod, C., Ellison, T. M., \& Fay, N. (2017). The sky is falling: Evidence of a negativity bias in the social transmission of information. Evolution \& Human Behavior, 38, 92-101. doi:10.1016/j.evolhumbehav.2016.07.004

Boyd, R., \& Richerson, P. J. (2006). Culture and the evolution of the human social instincts. In S. Levinson \& N. Enfield (Eds.), Roots of human sociality (pp. 453-477). Oxford, England: Berg.

Boyer, P., \& Parren, N. (2015). Threat-related information suggests competence: A possible factor in the spread of rumors. PLOS ONE, 1O(6), Article e0128421. doi:10.1371/ journal.pone.0128421

Carney, D. R., Jost, J. T., Gosling, S. D., \& Potter, J. (2008). The secret lives of liberals and conservatives: Personality profiles, interaction styles, and the things they leave behind. Political Psychology, 29, 807-840. doi:10.1111/j.1467-9221 .2008.00668.x

Cornelis, I., Van Hiel, A., Roets, A., \& Kossowska, M. (2009). Age differences in conservatism: Evidence on the mediating effects of personality and cognitive style. Journal of Personality, 77, 51-88. doi:10.1111/j.1467-6494.2008.00538.x

Crowson, H. M., Thoma, S. J., \& Hestevold, N. (2005). Is political conservatism synonymous with authoritarianism? The Journal of Social Psychology, 145, 571-592. doi:10.3200/ SOCP.145.5.571-592

Dodd, M. D., Balzer, A., Jacobs, C. M., Gruszczynski, M. W., Smith, K. B., \& Hibbing, J. R. (2012). The political left rolls with the good and the political right confronts the bad: Connecting physiology and cognition to preferences. Philosophical Transactions of the Royal Society B: Biological Sciences, 367, 640-649. doi:10.1098/rstb.2011.0268

Duckitt, J. (2001). A dual-process cognitive-motivational theory of ideology and prejudice. In M. P. Zanna (Ed.), Advances in experimental social psychology (Vol. 33, pp. 41-114). San Diego, CA: Academic Press.

Duckitt, J., Wagner, C., du Plessis, I., \& Birum, I. (2002). The psychological bases of ideology and prejudice: Testing 
a dual process model. Journal of Personality and Social Psychology, 83, 75-93. doi:10.1037/0022-3514.83.1.75

Federico, C. M., Hunt, C. V., \& Ergun, D. (2009). Political expertise, social worldviews, and ideology: Translating "competitive jungles" and "dangerous worlds" into ideological reality. Social Justice Research, 22, 259-279. doi:10.1007/ s11211-009-0097-0

Fessler, D. M. T. (2006). Steps toward the evolutionary psychology of a culture-dependent species. In P. Carruthers, S. Laurence, \& S. Stich (Eds.), The innate mind: Vol. 2. Culture and cognition (pp. 91-117). New York, NY: Oxford University Press.

Fessler, D. M. T., Holbrook, C., Pollack, J. S., \& Hahn-Holbrook, J. (2014). Stranger danger: Parenthood increases the envisioned bodily formidability of menacing men. Evolution \& Human Behavior, 35, 109-117. doi:10.1016/j.evolhum behav.2013.11.004

Fessler, D. M. T., Pisor, A. C., \& Navarrete, C. D. (2014). Negatively-biased credulity and the cultural evolution of beliefs. PLOS ONE, 9(4), Article e0095167. doi:10.1371/jour nal.pone.0095167

Graham, J., Haidt, J., \& Nosek, B. A. (2009). Liberals and conservatives rely on different sets of moral foundations. Journal of Personality and Social Psychology, 96, 10291046. doi:10.1037/a0015141

Hibbing, J. R., Smith, K. B., \& Alford, J. R. (2014). Differences in negativity bias underlie variations in political ideology. Behavioral \& Brain Sciences, 37, 297-307. doi:10.1017/ S0140525X13001192

Hilbig, B. E. (2009). Sad, thus true: Negativity bias in judgments of truth. Journal of Experimental Social Psychology, 45, 983-986. doi:10.1016/j.jesp.2009.04.012

Hilbig, B. E. (2012a). Good things don't come easy (to mind): Explaining framing effects in judgments of truth. ExperimentalPsychology, 59, 38-46. doi:10.1027/1618-3169/ a000124

Hilbig, B. E. (2012b). How framing statistical statements affects subjective veracity: Validation and application of a multinomial model for judgments of truth. Cognition, 125, 37-48. doi:10.1016/j.cognition.2012.06.009

Jones, J. (2016). Democrats more liberal on social issues than economic ones. Retrieved from http://www.gallup.com/ poll/191741/democrats-liberal-social-issues-economic-ones .aspx?g_source=liberal\&;g_medium=search\&g_campaign=tiles

Kemmelmeier, M. (2008). Is there a relationship between political orientation and cognitive ability? A test of three hypotheses in two studies. Personality and Individual Differences, 45, 767-772. doi:10.1016/j.paid.2008.08.003

Knoll, B. R., O’Daniel, T. J., \& Cusato, B. (2015). Physiological responses and political behavior: Three reproductions using a novel dataset. Research \& Politics, 2(4). doi:10.1177/ 2053168015621328

Kurzban, R. (2007). Representational epidemiology: Skepticism and gullibility. In S. W. Gangestad \& J. A. Simpson (Eds.), The evolution of mind: Fundamental questions and controversies (pp. 357-362). New York, NY: Guilford Press.

Lilienfeld, S. O., \& Latzman, R. D. (2014). Threat bias, not negativity bias, underpins differences in political ideology.
Behavioral \& Brain Sciences, 37, 318-319. doi:10.1017/ S0140525X1300263X

Mills, M., Gonzalez, F. J., Giuseffi, K., Sievert, B., Smith, K. B., Hibbing, J. R., \& Dodd, M. D. (2016). Political conservatism predicts asymmetries in emotional scene memory. Behavioural Brain Research, 306, 84-90. doi:10.1016/j.bbr 2016.03.025

Mills, M., Smith, K. B., Hibbing, J. R., \& Dodd, M. D. (2014). The politics of the face-in-the-crowd. Journal of Experimental Psychology: General, 143, 1199-1213. doi:10.1037/a0035177

Peer, E., Samat, S., Brandimarte, L., \& Acquisti, A. (2015). Beyond the Turk: An empirical comparison of alternative platforms for crowdsourcing online behavioral research. Retrieved from http://papers.ssrn.com/sol3/Papers.cfm? abstract_id=2594183

R Development Core Team. (2016). R: A language and environment for statistical computing (Version 3.3.1) [Computer software]. Retrieved from https://www.r-project.org/

Reed, A. E., Chan, L., \& Mikels, J. A. (2014). Meta-analysis of the age-related positivity effect: Age differences in preferences for positive over negative information. Psychology and Aging, 29, 1-15. doi:10.1037/a0035194

Rozin, P., \& Royzman, E. B. (2001). Negativity bias, negativity dominance, and contagion. Personality and Social Psychology Review, 5, 296-320. doi:10.1207/S15327957PSPR 0504_2

Sedek, G., Kossowska, M., \& Rydzewska, K. (2014). The importance of adult life-span perspective in explaining variations in political ideology. Behavioral E Brain Sciences, 37, 329330. doi:10.1017/S0140525X13002732

Stubbersfield, J. M., Tehrani, J. J., \& Flynn, E. G. (2015). Serial killers, spiders and cybersex: Social and survival information bias in the transmission of urban legends. British Journal of Psychology, 106, 288-307. doi:10.1111/bjop.12073

Sydell, L. (Writer). (2016, November 23). We tracked down a fake-news creator in the suburbs. Here's what we learned [Radio broadcast]. In C. Watson (Executive producer), All Things Considered. Retrieved from http://www.npr.org/ sections/alltechconsidered/2016/11/23/503146770/npr-findsthe-head-of-a-covert-fake-news-operation-in-the-suburbs

Trump, D. [realDonaldTrump]. (2016, August 29). Inner-city crime is reaching record levels. African-Americans will vote for Trump because they know I will stop the slaughter going on! [Tweet]. Retrieved from https://twitter.com/real donaldtrump/status/770247094439469058?lang=en

van Leeuwen, F., \& Park, J. H. (2009). Perceptions of social dangers, moral foundations, and political orientation. Personality and Individual Differences, 47, 169-173. doi:10.1016/j.paid.2009.02.017

Weeden, J., \& Kurzban, R. (2016). Do people naturally cluster into liberals and conservatives? Evolutionary Psychological Science, 2, 47-57. doi:10.1007/s40806-015-0036-2

White, M. P., Pahl, S., Buehner, M., \& Haye, A. (2003). Trust in risky messages: The role of prior attitudes. Risk Analysis, 23, 717-726. doi:10.1111/1539-6924.00350

Wilson, G. D., \& Patterson, J. R. (1968). A new measure of conservatism. British Journal of Social and Clinical Psychology, 7, 264-269. doi:10.1111/j.2044-8260.1968.tb00568.x 